\title{
Melatonin Entrains the Restored Circadian Activity Rhythms of Syrian Hamsters Bearing Fetal Suprachiasmatic Nucleus Grafts
}

\author{
Julia Grosse and Fred C. Davis \\ Department of Biology, Northeastern University, Boston, Massachusetts 02115
}

\begin{abstract}
A circadian pacemaker consists of at least three essential features: the ability to generate circadian oscillations, an output signal, and the ability to be entrained by external signals. In rodents, ablation of the suprachiasmatic nucleus (SCN) results in the loss of circadian rhythms in activity. Rhythmicity can be restored by transplanting fetal SCN into the brain of the lesioned animal, demonstrating the first two of the essential pacemaker features within the grafts. External signals, such as the light/dark cycle, have not, however, been shown to entrain the restored rhythms. Melatonin injections are an effective entraining stimulus in fetal and neonatal Syrian hamsters of the same developmental ages used to provide donor tissue for transplantation. Therefore, melatonin was used to test the hypothesis that SCN grafts contain an entrainable pacemaker. Daily injections of melatonin were given to $\mathrm{SCN}$-lesioned hosts
\end{abstract}

Mammalian circadian rhythms are regulated by a pacemaker within the suprachiasmatic nucleus (SCN) of the hypothalamus (Klein et al. 1991). In rats and Syrian hamsters ablation of the SCN abolishes circadian activity and rest rhythms, which can be restored by transplantation of fetal SCN tissue (Drucker-Colin et al., 1984; Lehman et al., 1987; DeCoursey and Buggy, 1988). In addition, the period of the restored rhythm is characteristic of the donor's genotype (Ralph et al., 1990), demonstrating that the transplanted tissue contains the circadian timing mechanism. Although these studies have demonstrated the effectiveness of transplants in restoring activity rhythms, and in one case a humoral rhythm (Earnest et al., 1989), neither the entrainment of restored rhythms by light nor the restoration of photoperiodic responses has been observed (Lehman et al., 1987; Matsumoto et al., 1996). Thus it has not been known whether transplanted SCN retains the ability to be entrained. It is possible that cells capable of generating circadian oscillations and producing an output signal survive transplantation but that cells or mechanisms required for entrainment do not.

The possibility that melatonin might serve as an entraining signal for SCN grafts was suggested by the finding that prenatal injections to pregnant hamsters entrained the activity rhythms expressed by the pups after weaning (Davis and Mannion, 1988). More recently, daily melatonin injections given directly to newborn hamsters were also shown to cause entrainment (Grosse et al., 1996). These findings, together with evidence for melatonin

\footnotetext{
Received May 26, 1998; revised July 16, 1998; accepted July 17, 1998.

This work was supported by National Institutes of Health Grant HD18686 to F.C.D.

Correspondence should be addressed to Fred C. Davis, Department of Biology, 414 Mugar Life Sciences Building, Northeastern University, Boston, MA 02115.

Copyright (C) 1998 Society for Neuroscience $\quad 0270-6474 / 98 / 188032-06 \$ 05.00 / 0$
}

beginning on the day after transplantation of fetal SCN. Two groups that received melatonin at different times of day $12 \mathrm{hr}$ apart each showed significantly clustered phases but with average phases that differed by $8.67 \mathrm{hr}$. Thus melatonin was able to entrain the restored circadian activity rhythms. In contrast to these initial injections, injections given 6 weeks after transplantation were unable to entrain or phase shift the rhythms. The results demonstrate that $\mathrm{SCN}$ grafts contain an entrainable circadian pacemaker. In addition, the results also indicate that the fetal SCN is directly sensitive to melatonin and, as with intact hamsters, sensitivity to melatonin is lost during SCN development.

Key words: suprachiasmatic nucleus; graft; circadian rhythm; transplantation; pineal; melatonin receptors in the fetal and newborn hamster SCN (Duncan and Davis, 1993), suggest that the fetal and newborn hamster SCN are targets for melatonin. In transplantation studies, $\mathrm{SCN}$ from either fetuses or newborn hamsters are transplanted. Thus, if the direct action of melatonin on the intact fetal and neonatal SCN is sufficient to cause entrainment, and an entrainable pacemaker is present in grafts, then melatonin should entrain rhythms restored by transplanted SCN. A preliminary study, which examined the effects of melatonin on hamsters with SCN grafts, in fact suggested that rhythms restored by transplantation could respond to an external entraining cue (Romero and Silver, 1989). Other evidence indicated that the rhythmicity of an intact host can influence metabolic activity within grafts (Servière et al., 1994).

The first aim of the present study was to determine whether melatonin injections could entrain the activity rhythms of hamsters bearing newly implanted SCN grafts. Second, because the ability of melatonin to entrain the rhythms of neonatal hamsters is lost after postnatal day 5 (P5) (Grosse et al., 1996), and melatonin binding site density falls during postnatal development (Duncan and Davis, 1993), the effect of melatonin injections on more established grafts was also examined. If the ability of melatonin to entrain the SCN disappears during the maturation of grafts, this would suggest that the loss during normal development is part of a program intrinsic to the grafted tissue rather than to changes that normally occur elsewhere in the developing animal, such as the appearance of the pup's own pineal melatonin rhythm (Rollag and Stetson, 1981).

\section{MATERIALS AND METHODS}

Animals. Adult (8-week-old) male and female Syrian hamsters (Mesocricetus auratus) were obtained from Charles River Laboratories (Kingston, NY) and maintained on a 14/10 hr light/dark cycle (lights off at $1600 \mathrm{hr}$ Eastern Standard Time) with food and water available ad 
libitum. To obtain fetal tissue for transplantation, estrous cycles of females were monitored by daily examination of the vaginal discharge, and animals were mated overnight on the night of ovulation. Fertilization was assumed to have occurred midway through the dark portion of the light/dark cycle, and the $24 \mathrm{hr}$ after mating was designated embryonic day 1 (E1). To ensure that the donor fetuses received no circadian cues before transplantation, pregnant females were transferred to dim constant light (dim LL, <2 lux) at the time of lights off on E1 and on E7 received electrolytic lesions of the SCN (see Surgery).

Activity recording. Activity rhythms were monitored in dim LL by housing animals individually in cages equipped with a running wheel. Wheel revolutions were recorded by closure of a microswitch mounted on the cage and collected and stored in 10 min bins using Datacol 3 software (Minimitter, Sunriver, OR). Display and analysis of activity recordings were performed using Circadia software (Behavioral Cybernetics, Cambridge, MA). Estimates of free-running period to a resolution of $\pm 5 \mathrm{~min}$ were made by adjusting the folding period of the actogram until vertical alignment of successive activity bouts was achieved.

Surgery. Pregnant females and both male and female host animals received electrolytic lesions of the SCN. Animals were anaesthetized with sodium pentobarbital $(100 \mathrm{mg} / \mathrm{kg})$ and placed in a stereotaxic frame. An electrode (platinum iridium, OD insulated except for $0.3 \mathrm{~mm}$ at the tip) was lowered at the midline to a point $8.3 \mathrm{~mm}$ below the skull surface and $0.6 \mathrm{~mm}$ anterior to bregma (incisor bar $2 \mathrm{~mm}$ below ear bar). With a subcutaneous cathode, a current of $4 \mathrm{~mA}$ was passed for $10 \mathrm{sec}$. Pregnant females were then returned to dim LL until transplantation on E14. Recipient animals were also transferred to dim LL and housed individually in cages equipped with a running wheel for recording of activity rhythms. Any animal that subsequently displayed a rhythmic pattern of activity and rest was removed from the study.

Two to 3 weeks after receiving SCN lesions, SCN-lesioned hosts received grafts of hypothalamic tissue obtained from fetuses collected on E14. Pregnant hamsters received a lethal dose of sodium pentobarbital, their fetuses were rapidly removed and decapitated, and the brains were dissected into Geys balanced salt solution (Life Technologies, Grand Island, NY). The fetal brains were then transferred to $67 \%$ Eagle's basal medium and 33\% Earle's balanced salt solution at room temperature. A block of tissue containing the optic chiasm and SCN region of the hypothalamus was dissected from the brains and used for transplantation within $90 \mathrm{~min}$.

Recipients of grafts were anaesthetized and placed in the stereotaxic frame. A glass micropipette (Wiretrol II; Drummond Scientific, Broomall, PA) was used to take up one block of donor tissue containing both SCN from a single fetus (a volume of $<1 \mu \mathrm{l}$ ) and was lowered through the hole previously drilled for the SCN lesion to a point $\sim 8 \mathrm{~mm}$ below the dural surface. The plunger was held in place while the pipette was withdrawn, leaving the graft in the brain. Animals were returned to wheel-running cages in dim LL and activity recording continued. In all, five or six fetuses from eight litters were used (44 transplants in total).

Protocol. Of the host animals that received SCN lesions, 44 (75\%) were arrhythmic and received grafts (15 females and 29 males). A total of eight donor litters were used, with four different donor litters contributing tissue to each treatment group. Hosts were divided into two treatment groups. Group 1 (6 females and 16 males) received melatonin injections (25 $\mu \mathrm{g}$ in $200 \mu \mathrm{l}$ of $0.1 \%$ ethanolic saline) at $0900 \mathrm{hr}$ and vehicle $(0.1 \%$ ethanolic saline) injections at $2100 \mathrm{hr}$ for $7 \mathrm{~d}$ beginning on the day after transplantation. Group 2 ( 9 females and 13 males) received vehicle injection at $0900 \mathrm{hr}$ and melatonin injection at $2100 \mathrm{hr}$ daily for the $7 \mathrm{~d}$ after transplantation. After injections were completed, the hamsters were left undisturbed while activity recording continued for another 5 weeks. Hosts that had not shown restoration of robust circadian rhythmicity by this time were removed from the experiment. Ultimately, both groups consisted of 12 hamsters, with 4 females and 8 males in each. Included in the final groups were hosts that had received tissue from each of the eight donor litters; i.e., all donor litters contributed viable grafts to the study.

To examine the response to melatonin and vehicle injection in hosts with grafts that had become well established, the animals that showed the most robust rhythms 5 weeks after transplantation received a second set of injections. Hamsters received injections of either melatonin $(n=6 ; 3$ from each of groups 1 and 2$)$ or vehicle $(n=5 ; 3$ from group 1 and 2 from group 2) at $1100 \mathrm{hr}$ for 5 consecutive days. The injections fell at different circadian times in different animals (see Results). Activity recording was continued for a further $10 \mathrm{~d}$ after the final injection to assess any phase shift that may have occurred. All animals were then perfused, and the brains were processed for immunocytochemical detection of vasoactive intestinal polypeptide (VIP).

Data analysis and statistics. The phase of activity onset of restored rhythms was determined for the first day after a set of injections. For the initial set of injections phase was assessed by three observers (two with no knowledge of the experimental groups) with eye fit lines through at least 3 weeks of activity onsets. The eye fit lines were extrapolated back to the day after the last injection. In all cases, the estimates of phase were within $2 \mathrm{hr}$ of each other. Where estimates differed, the median of the three estimates was used. Phases were plotted on a circle representing the $24 \mathrm{hr}$ of the day after the last injection. The average phase for a given group was calculated by vector addition, with the length of the average vector, $r$, representing the scatter among phases. The Rayleigh test was used to determine whether the distribution of phases within a group was significantly different from uniform, and the Mardia-Watson-Wheeler test determined whether there was a difference between the distribution of the two groups (Batschelet, 1972). Any phase shift and/or entrainment caused by the injections given after the establishment of restored rhythms was assessed by comparing actual and predicted onsets (determined by eye fit lines) on the day after the last of the five injections.

Immunocytochemistry. The extent of SCN lesions and the presence of $\mathrm{SCN}$ tissue within grafts were assessed at the end of the experiment by immunoreactivity for vasoactive intestinal polypeptide (VIP-IR). Animals received an overdose of sodium pentobarbital and were perfused intracardially with $0.01 \mathrm{M}$ PBS containing 25,000 U/1 heparin, followed by $4 \%$ paraformaldehyde in $0.01 \mathrm{M}$ PBS (PFA). Brains were removed and post-fixed for $2 \mathrm{hr}$ in PFA and then cryoprotected overnight in $20 \%$ sucrose-PBS. Sixty micrometer coronal sections through the graft and lesion sites were cut on a freezing microtome, and free-floating sections were then processed for VIP-IR. Sections were treated for $10 \mathrm{~min}$ in $0.5 \% \mathrm{H}_{2} \mathrm{O}_{2}$ and then rinsed in $0.01 \mathrm{M}$ PBS and incubated overnight at $4^{\circ} \mathrm{C}$ in $0.01 \mathrm{M}$ PBS, $1 \%$ bovine serum albumen, and $0.3 \%$ Triton $\mathrm{X}-100$ containing anti-VIP antibody (1:5000; Peninsula Laboratories, Belmont, CA) and $2 \%$ normal goat serum. Detection of primary antiserum was achieved using the Vectastain Elite avidin-biotin complex method (Vector Laboratories) and visualized using diaminobenzidine.

\section{RESULTS}

Restoration of circadian rhythmicity sufficiently robust to assess phase of activity onset was achieved in $54.5 \%$ of hosts $(55.2 \%$ of males and $53.3 \%$ of females). The mean period \pm SEM of restored rhythms was $24.05 \pm 0.04 \mathrm{hr}$ (24.06 hr in males and 24.04 hr in females). Figures 1 and 2 show examples of actograms from two animals from each of the experimental groups. Histological assessment confirmed that animals that were arrhythmic after SCN lesion had no VIP-IR in the SCN region of the hypothalamus. Additionally, the brains of all animals that showed restoration of circadian rhythms had VIP-IR graft tissue, generally located in the anterior hypothalamus, above or within the third ventricle. Figure 3 shows photomicrographs of lesions and grafts from hosts in both experimental groups.

The phases of activity onset on the day after the first set of injections are shown in Figure 4. The mean phase of activity onset of the hamsters in group 1 was $0030 \mathrm{hr}(8.5 \mathrm{hr}$ before the time of melatonin injection at $0900 \mathrm{hr}$ ), and that of Group 2 was $0910 \mathrm{hr}$ (11.83 hr before the time of melatonin injection at $2100 \mathrm{hr}$ ), a difference of $8.67 \mathrm{hr}$. The distribution of phases for each group was significantly clustered (group $1, r=0.67$; group $2, r=0.89$; $p<0.01$ in both groups, Rayleigh test). There was also a significant difference between the distributions of the two groups $(p<$ 0.01, Mardia-Watson-Wheeler test). Thus melatonin injections given at opposite times of day to hamsters bearing newly implanted SCN grafts were able to entrain the restored rhythms to different phases.

In 10 of the 11 hosts tested, a second series of melatonin or vehicle injections delivered 6 weeks after transplantation failed to induce either phase shifts or entrainment to the injections (Figs. 1 and 2). The circadian times (CTs, with activity onset as CT 12) 
A

Figure 1. Double-plotted actograms of two hamsters that received melatonin injections in the morning (circles) and vehicle (diamonds) in the evening (group 1) for $7 \mathrm{~d}$ immediately after transplantation $(T)$. The hamsters also received injections (as indicated) 6 weeks after transplantation. The heavy vertical lines are the eye fit lines used to estimate the phases of activity onset on the day after the initial set of injections (top end of the line). Asterisks on the time scales at the tops of the actograms also indicate the phases. Eye fit lines before and after the later set of injections were used to assess the effects of the injections. In most cases these two lines appear as a single line, because no shifts or changes in period occurred. The VIP-immunoreactive grafts of animals $\mathrm{A}$ and $\mathrm{B}$ are shown in Figure 3, $A$ and $B$.

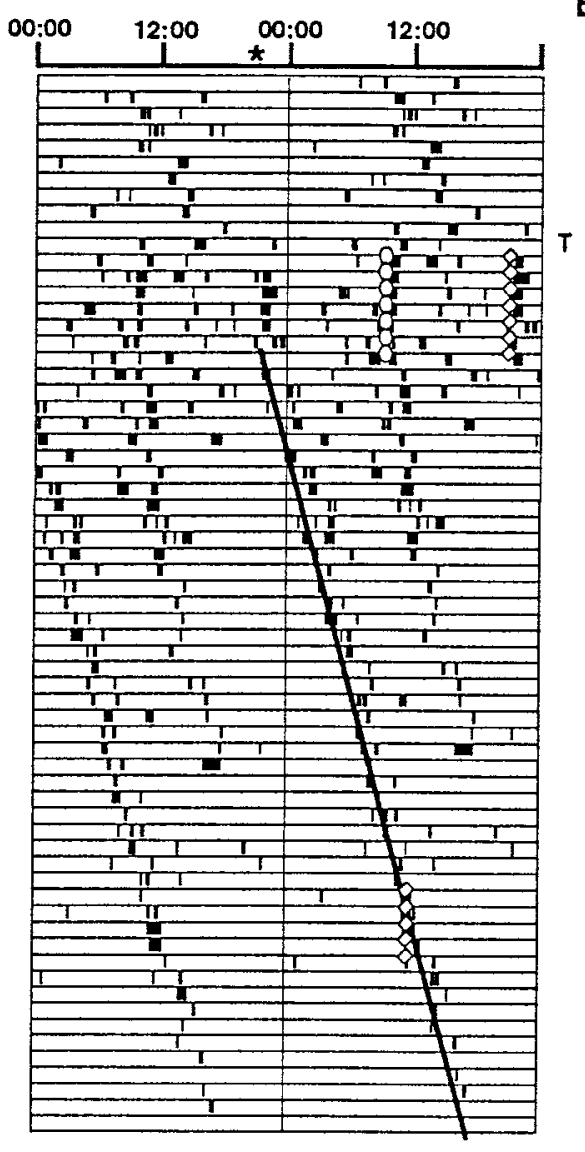

B

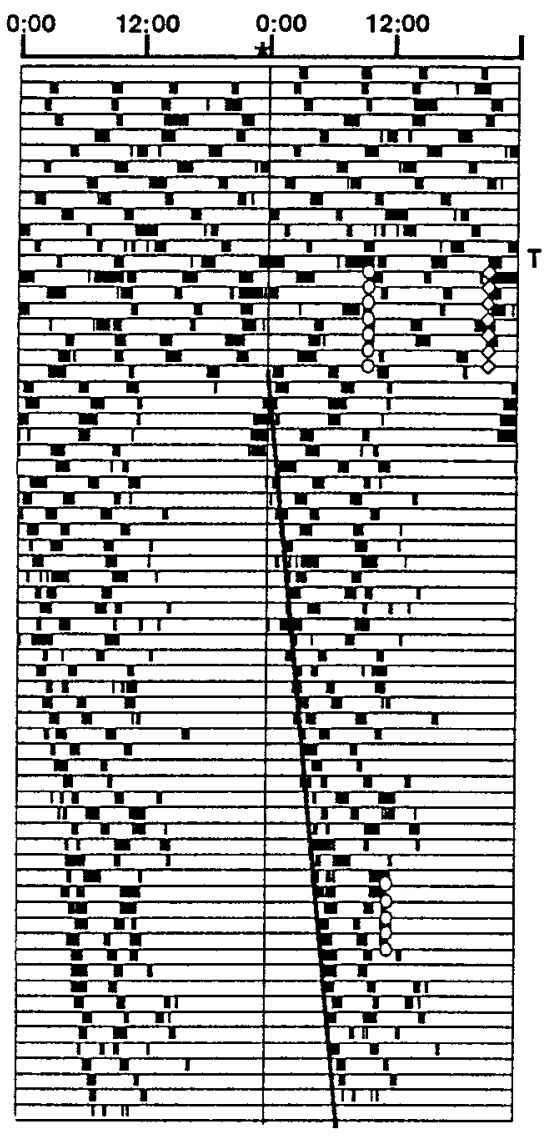

at which the first of the five injections occurred for the hamsters that received melatonin were $9,17,18,22,23$, and 24 , and for those that received vehicle were $6,11,12,17$, and 21.5 . The only hamster that showed a phase shift did so in response to vehicle injections at CT 11 (Fig. 2B). Thus melatonin did not entrain or phase shift the activity rhythms of hamsters with well-established SCN grafts.

\section{DISCUSSION}

Injections of melatonin to SCN-lesioned adult hamsters bearing newly implanted fetal SCN grafts entrained the restored circadian activity rhythms. In particular, significantly different average phases were established by injections given at different times of day. Melatonin must have caused the difference in average phase, because the hosts and the donor tissues were all treated identically in the two groups except for the timing of melatonin exposure; all hosts received injections twice a day, and all donor tissues came from fetuses of SCN-lesioned dams that were maintained under the same conditions.

Entrainment of the restored rhythms with injections given immediately after transplantation indicates that circadian oscillations were being generated during this time, within $7 \mathrm{~d}$ of E14, the age of the tissue when transplanted. The results also suggest that the fetal and neonatal hypothalami are direct targets of melatonin. It seems unlikely that synaptic communication between potential melatonin targets within the host (Morgan et al., 1994) and the graft would have been established within $7 \mathrm{~d}$ after transplantation. Furthermore, although the presence of melatonin receptors within grafts has not been examined, the presence of receptors in intact fetal and neonatal SCN (Duncan and Davis, 1993) suggests that receptors would be present in grafts. It cannot be excluded, however, that a humoral signal from the host to the graft mediates indirect effects of the injections. In addition, although the dose of melatonin used $(25 \mu \mathrm{g})$ is commonly used in reproductive studies, such an injection produces serum levels three orders of magnitude above physiological levels (Maywood et al., 1993). Thus it is not known whether the entrainment observed here is a physiological effect of melatonin.

In contrast to the effects of injections given immediately after transplantation, a second set of injections given 6 weeks after transplantation had no measurable effect on the phase of the rhythms. Although it is possible that melatonin injections given over $>5 \mathrm{~d}$ could at some phases cause entrainment, any such effect on established grafts would have to be much smaller than the effects on perinatal grafts. That is, it is likely that the melatonin injections given immediately after transplantation fell at a wide range of phases of the oscillations generated within the grafts; melatonin was given at two times of day, and the phases of oscillations within different grafts were likely to have been initially scattered, because they came from fetuses of SCN-lesioned dams (Davis and Gorski, 1988). Despite this wide range of initial phases, the groups became clustered after only $7 \mathrm{~d}$ of injections. Thus in at least some of the grafts large phase shifts must have been produced. In contrast, no phase shifts in response to five melatonin injections were observed when given 6 weeks after transplantation.

The apparent loss in sensitivity to melatonin in grafts is consistent with a loss observed in hamster pups; entrainment of hamster pups can be achieved with injections on P1-P5 but not 
A

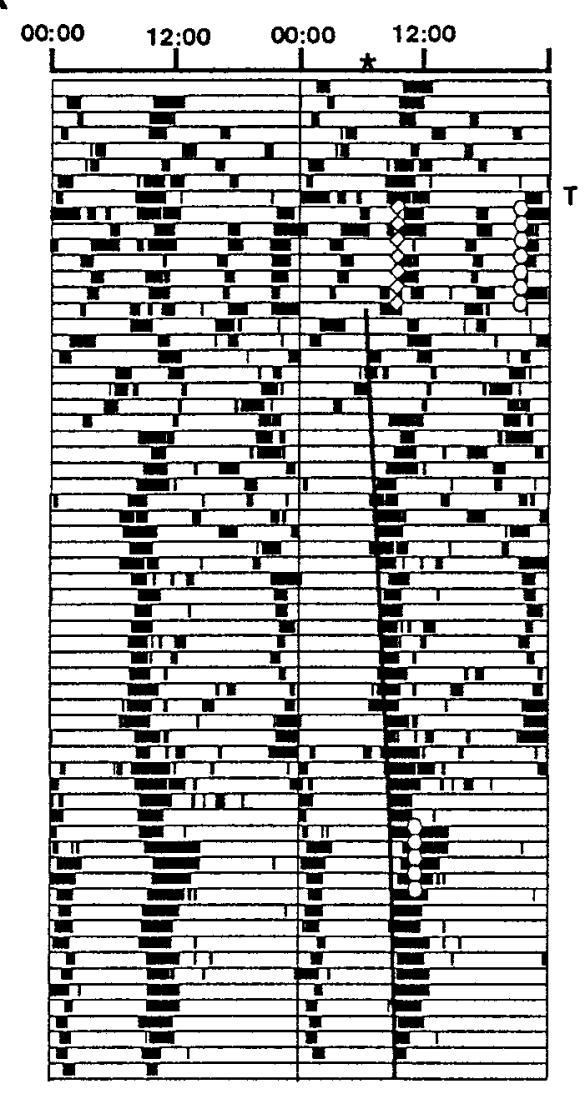

B

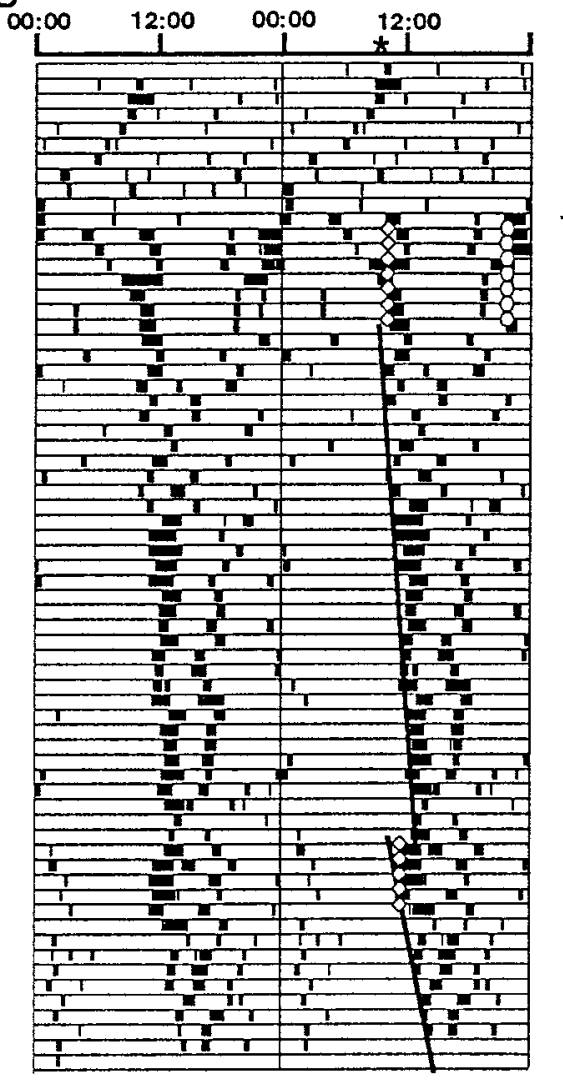

Figure 2. Double-plotted actograms of two hamsters that received melatonin injections in the evening (circles) and vehicle (diamonds) in the morning (group 2) for 7 days immediately after transplantation $(T)$. The hamsters also received injections (as indicated) 6 weeks after transplantation. The heavy vertical lines are the eye fit lines used to estimate the phases of activity onset on the day after the initial set of injections (top end of the line). Asterisks on the time scales at the tops of the actograms also indicate the phases. Eye fit lines before and after the later set of injections were used to assess the effects of the later injections. Record $B$ shows an advance phase shift in response to vehicle injections. The VIP-immunoreactive grafts of animals $\mathrm{A}$ and $\mathrm{B}$ are shown in Figure $3, C$ and $D$. with injections on P6-P10 (Grosse et al., 1996). It is possible that the normal loss in sensitivity to melatonin is part of a developmental program intrinsic to the SCN; the loss occurred in the grafts despite isolation from the rest of the developing animal. Plausible hypotheses for the loss of sensitivity in intact pups, such as synaptogenesis of retinal afferents or the onset of the pup's own pineal melatonin rhythm, are less plausible, because neither of these would have occurred for the grafts. On the other hand, other afferents from the host are likely to innervate the grafts and could influence its development in ways similar to what might occur during normal development (Lehman et al., 1987; Botchkina and Morin, 1993, 1995; Ueda et al., 1995, 1996).

The average phase of entrainment achieved by melatonin injections in this study differed from that seen after injections to neonatal hamsters (Grosse et al., 1996). In neonates, the average phase of activity onset after weaning was almost coincident with the time at which the melatonin injections were given. The age of the neonates in that study was P1-P5 during the injections, similar to the age of the grafts in the present study (approximately E15-P6). However, in the present study, the average phase (both groups combined) occurred $10 \mathrm{hr}$ before the injection so that the melatonin was closer to being coincident with the end of activity rather than the onset of activity. The reason for this difference is unclear. The dose of melatonin used in the current study was the same per gram of body weight as that used by Grosse et al. (1996), and because melatonin distributes rapidly through tissues, it is unlikely that there would have been a lag of several hours before the melatonin reached the grafts. A better explanation may be that melatonin actually entrains the pacemaker in the same way in both situations, but that the phase relationship between the pacemaker within the SCN and the expressed activity is different. For example, if in one situation activity onset was linked to CT 12 (intact pups) but in the other to CT 0 (graft/host), then the expressed rhythms would have opposite phases. Rhythms restored by transplantation often show bimodal patterns in which it is ambiguous as to which of two opposite phases should be considered activity onset (Lehman et al., 1987; Davis and Viswanathan, 1996). It is possible that abnormal communication between the grafted SCN and the host brain target tissues could affect the relationship between pacemaker output signals and the response of downstream systems. For example, the output of grafts may depend entirely on humoral signals (Silver et al., 1996), whereas SCN output in intact hamsters might include specific synaptic connectivity. Interestingly, abnormal communication by grafts would not, in this hypothesis, simply introduce variability in phase or ambiguity between two possible choices but would have to be consistently different in some way so that similar phases among the restored rhythms of a particular treatment group are observed.

Because in the present study activity onset of the restored rhythms was closer to coinciding with the time of the vehicle-only injections than with the time of the melatonin injections, it is tempting to suggest that the vehicle, not melatonin, caused the entrainment. This, however, cannot be. Both injection times included vehicle, and although it is possible that the vehicle has an effect that is either enhanced or reduced by melatonin, melatonin must have had an effect that was responsible for the phase difference between the experimental groups. Furthermore, in a previous study by Grosse et al. (1996), pups received single injections of either melatonin or vehicle only each day, and the vehicle-only injections did not cause entrainment.

The lack of entrainment or phase shifts after the second set of 

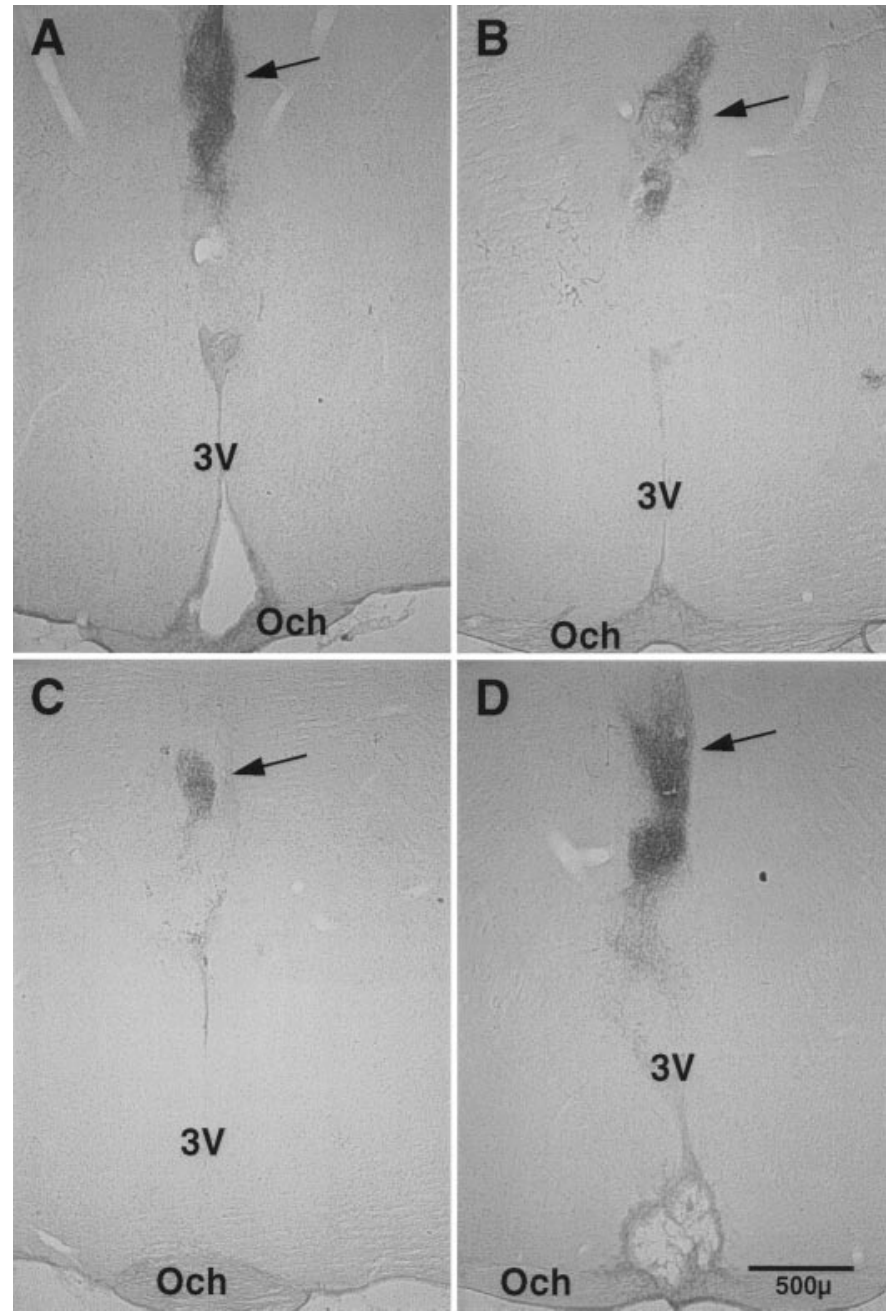

Figure 3. Photomicrographs of coronal sections that contained grafted tissue with VIP immunoreactivity (arrows) in four of the hamsters from the study. Lesions of the host SCN can also be seen in $A$ and $D$. Och, Optic chiasm; $3 V$, third ventricle.

melatonin injections suggests that an established SCN graft behaves in a manner similar to the mature, intact SCN despite the abnormal environment in which it developed. Intact adult hamsters will show advance phase shifts in response to a melatonin or vehicle-only injection when the injection is given at approximately CT 9-11 (Mead et al., 1992), but there is no specific effect of melatonin over and above that of vehicle only (Hastings et al., 1992). The only hamster in the present study that showed any response to the second set of injections was injected with vehicle at CT 11 and showed a phase advance. This suggests that hamsters bearing grafts can respond to nonphotic stimuli in a manner similar to that of intact animals. Although Matsumoto et al. (1996) did not see a robust effect of the benzodiazepene triazolam on the restore rhythms of hamsters with SCN grafts, a small number of animals did show phase shifts. Further studies are needed to firmly establish whether mature grafts can respond to a nonphotic stimulus.

Overall, the present results demonstrate that an SCN graft includes three essential features of a circadian pacemaker: circadian timing, an output signal, and the ability to become entrained. In addition, the results support the hypothesis that melatonin is involved in communicating time-of-day information from the

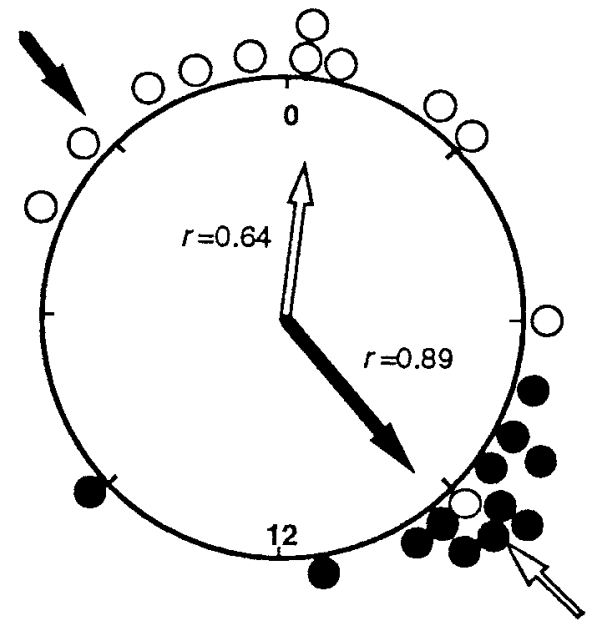

Figure 4. Phases of activity onset on the day after the final injection for all animals plotted on a circle representing the $24 \mathrm{hr}$ of that day. Open circles represent individual animals that received melatonin in the morning and vehicle in the evening (group 1), and closed circles represent animals that received vehicle in the morning and melatonin in the evening (group 2). The average phase for each group is shown by the arrows within the circle (open arrow, group 1; closed arrow, group 2), and the degree of synchrony within each group is represented by the length of the arrows and by the value of $r$. Arrows outside the circle indicate the times of the injections.

mother to the fetus. The results indicate that the fetal hamster $\mathrm{SCN}$ is a direct target for entrainment by melatonin and that this sensitivity to melatonin is transient, disappearing as a result of developmental changes intrinsic to the SCN. In addition to the entrainment demonstrated by these results, melatonin might also have an effect on SCN development. Rhythms appeared to have been restored more rapidly in the present study than in previous studies from this laboratory, and a similar observation was made in a preliminary study from another laboratory (Romero and Silver, 1989). In the present study, the appropriate direct comparisons to address this question were not available, and the question of whether melatonin has an organizational effect on the developing SCN, in addition to entrainment, will require further studies.

\section{REFERENCES}

Batschelet E (1972) Recent statistical methods for orientation data. In: Animal Orientation and Navigation (Galler SR, ed), pp 61-91. Washington, DC: US Government Printing Office.

Botchkina GI, Morin LP (1993) Development of the hamster serotoninergic system: cell groups and diencephalic projections. J Comp Neurol 338:405-431.

Botchkina GI, Morin LP (1995) Organization of permanent and transient neuropeptide Y-immunoreactive neuron groups and fiber systems in the developing hamster diencephalon. J Comp Neurol 357:573-602.

Davis FC, Gorski RA (1988) Development of hamster circadian rhythms: role of the maternal suprachiasmatic nucleus. J Comp Physiol [A] 162:601-610.

Davis FC, Mannion J (1988) Entrainment of hamster pup circadian rhythms by prenatal melatonin injections to the mother. Am J Physiol 255:R439-R448.

Davis FC, Viswanathan N (1996) The effect of transplanting one or two suprachiasmatic nuclei on the period of the restored rhythm. J Biol Rhythms 11:291-301.

DeCoursey PJ, Buggy J (1988) Restoration of circadian locomotor activity in arrhythmic hamsters by fetal SCN transplants. Comp Endocrinol 7:49-54.

Drucker-Colin R, Aguilar-Roblero R, Garcia-Hernandez F, Fernandez- 
Cancino F, Rattoni FB (1984) Fetal suprachiasmatic nucleus transplants: diurnal rhythm recovery of lesioned rats. Brain Res 311:353-357.

Duncan MJ, Davis FC (1993) Developmental appearance and age related changes in specific $2-\left[{ }^{125} \mathrm{I}\right]$ iodomelatonin binding sites in the suprachiasmatic nuclei of female Syrian hamsters. Dev Brain Res 73:205-212.

Earnest DJ, Sladek CD, Gash DM, Wiegand SJ (1989) Specificity of circadian function in transplants of the fetal suprachiasmatic nucleus. J Neurosci 9:2671-2677.

Grosse J, Velickovic A, Davis FC (1996) Entrainment of Syrian hamster circadian activity rhythms by neonatal melatonin injections. Am J Physiol 270:R533-R540.

Hastings MH, Mead SM, Vindlacheruvu RR, Ebling FJP, Maywood ES, Grosse J (1992) Non-photic phase shifting of the circadian activity rhythm of Syrian hamsters: the relative potency of arousal and melatonin. Brain Res 591:20-26.

Klein DC, Moore RY, Reppert SM (1991) Suprachiasmatic nucleus: the mind's clock. New York: Oxford UP.

Lehman MN, Silver R, Gladstone WR, Kahn RM, Gibson M, Bittman EL (1987) Circadian rhythmicity restored by neural transplant. Immunocytochemical characterization of the graft and its integration with the host brain. J Neurosci 7:1626-1638.

Matsumoto S, Basil J, Jetton AE, Lehman MN, Bittman EL (1996) Regulation of the phase and period of circadian rhythms restored by suprachiasmatic transplants. J Biol Rhythms 11:145-162.

Maywood EL, Hastings MH, Max M, Ampleford E, Menaker M, Loudon ASI (1993) Circadian and daily rhythms of melatonin in the blood and pineal gland of free-running and entrained Syrian hamsters. J Endocrinol 136:65-73.
Mead SM, Ebling FJP, Maywood ES, Humby T, Herbert J, Hastings MH (1992) A non-photic stimulus causes instantaneous phase-advances of the light-entrainable circadian oscillator of the Syrian hamster, but does not induce the expression of c-fos in the suprachiasmatic nuclei. J Neurosci 12:2516-2522.

Morgan PJ, Barrett P, Howell HE, Helliwell R (1994) Melatonin receptors: Localization, molecular pharmacology and physiological significance. Neurochem Int 24:101-146.

Ralph MR, Foster RG, Davis FC, Menaker M (1990) Transplanted suprachiasmatic nucleus determines circadian period. Science 247:975-978.

Rollag MD, Stetson MH (1981) Ontogeny of the pineal melatonin rhythm in golden hamsters. Biol Reprod 24:311-314.

Romero M-T, Silver R (1989) Control of phase and latency to recover circadian locomotor rhythmicity following transplantation of fetal SCN into lesioned adult hamsters. Soc Neurosci Abstr 15:725.

Servière J, Gendrot G, LeSauter J, Silver R (1994) Host resets phase of grafted suprachiasmatic nucleus: A 2-DG study of time course of entrainment. Brain Res 655:168-176.

Silver R, LeSauter J, Tresco PA, Lehman MN (1996) A diffusible coupling signal from the transplanted suprachiasmatic nucleus controlling circadian locomotor rhythms. Nature 382:810-813.

Ueda S, Matsumoto Y, Nisimura A, Azmitia EC, Kawata M (1995) Role of neuropeptide $\mathrm{Y}$ projection on the development of serotonergic innervation in the suprachiasmatic nucleus of the rat, shown by triple intraocular grafts. Brain Res 673:325-330.

Ueda S, Aikawa M, Ishizuya-Oka A, Nishimura A, Kawata M (1996) Alteration of serotonergic innervation in the suprachiasmatic nucleus of the rat following removal of input fibers from retina and lateral geniculate nucleus. Neurosci Lett 211:97-100. 